\title{
A Review of Risk Factors for Cognitive Impairment in Stroke Survivors
}

\author{
Mohd Faizal Mohd Zulkifly, ${ }^{1,2}$ Shazli Ezzat Ghazali, ${ }^{1}$ Normah Che Din, ${ }^{1}$ \\ Devinder Kaur Ajit Singh, ${ }^{3}$ and Ponnusamy Subramaniam ${ }^{1}$
}

\author{
${ }^{1}$ Health Psychology Programme, School of Healthcare Sciences, Universiti Kebangsaan Malaysia, \\ Jalan Raja Muda Abdul Aziz, 50300 Kuala Lumpur, Malaysia \\ ${ }^{2}$ Center for Neuroscience Services \& Research (P3Neuro), Universiti Sains Malaysia (USM), \\ Health Campus, 16150 Kubang Kerian, Kelantan, Malaysia \\ ${ }^{3}$ Physiotherapy Programme, School of Rehabilitation Sciences, Universiti Kebangsaan Malaysia, \\ Jalan Raja Muda Abdul Aziz, 50300 Kuala Lumpur, Malaysia
}

Correspondence should be addressed to Ponnusamy Subramaniam; ponnusaami@gmail.com

Received 10 January 2016; Accepted 4 May 2016

Academic Editor: Helen F. K. Chiu

Copyright (C) 2016 Mohd Faizal Mohd Zulkifly et al. This is an open access article distributed under the Creative Commons Attribution License, which permits unrestricted use, distribution, and reproduction in any medium, provided the original work is properly cited.

\begin{abstract}
In this review, we aimed to identify the risk factors that may influence cognitive impairment among stroke survivors, namely, demographic, clinical, psychological, and physical determinants. A search from Medline, Scopus, and ISI Web of Science databases was conducted for papers published from year 2004 to 2015 related to risk factors of cognitive impairment among adult stroke survivors. A total of 1931 articles were retrieved, but only 27 articles met the criteria and were reviewed. In more than half of the articles it was found that demographical variables that include age, education level, and history of stroke were significant risk factors of cognitive impairment among stroke survivors. The review also indicated that diabetes mellitus, hypertension, types of stroke and affected region of brain, and stroke characteristics (e.g., size and location of infarctions) were clinical determinants that affected cognitive status. In addition, the presence of emotional disturbances mainly depressive symptoms showed significant effects on cognition. Independent relationships between cognition and functional impairment were also identified as determinants in a few studies. This review provided information on the possible risk factors of cognitive impairment in stroke survivors. This information may be beneficial in the prevention and management strategy of cognitive impairments among stroke survivors.
\end{abstract}

\section{Introduction}

It has been reported that approximately 15 to $30 \%$ of stroke survivors live with permanent disability [1]. This includes physical, social, and cognitive functions [2-4]. Various domains of cognitive function such as attention, concentration, memory, social cognition, language, spatial and perceptual skills, and higher-order executive functions may be affected in stroke survivors [5]. The most common cognitive impairments among stroke survivors are memory, orientation, language and attention [6], executive dysfunction (initiation inhibition, mental flexibility), and aphasia $[7,8]$. Some of the consequences of cognitive impairments are high prevalence of morbidity and mortality, increased length of hospital stay, increased number of admissions to rehabilitation centre, dependency in ADL, and reduced functional outcomes [9-11].

Cognitive deficits among stroke survivals are widely recognized research area. However, the risk factors for cognitive impairment among stroke survivors have not been addressed adequately [12]. Previous literature reviews predominantly explain vascular risk factors and mechanisms of cognitive impairments [12, 13], impact and treatment options for cognitive impairment [14], and interaction of vascular risk factors with stroke [15], and overlooked or little emphasis was given on the psychological and physical determinants. This may be due to difference in studied variables, methodologies, and outcome measurements. Most of the studies focused on 
modifiable variables such as sociodemographic and clinical factors. Education level, level of alcohol use, smoking status, dietary intake, atrial fibrillation, and cerebral microbleeds are some of the factors that could be modified to prevent poststroke cognitive decline [16, 17]. Physical activity has been reported to be a protective factor to prevent the risk for cognitive impairment and vascular dementia among older adults with white matter changes [18]. Stroke survivors with vascular cognitive impairment who attended day care rehabilitation were reported to benefit from physical activity and exercise. Exercise is advocated to heighten cognitive function among stroke survivors [19].

Stroke survivors with cognitive impairment are most likely to be dependent in activities of daily living. Further deterioration is possible as a result of limitation in activities. Psychiatric problems are also common among stroke survivors. This may delay recovery process and further impair cognitive function due to adaptations to unhealthy lifestyles or noncompliance to rehabilitation [20]. Prevalence of moderate to severe depression and anxiety was found to be $22.8 \%$ and $21.1 \%$, respectively, in stroke survivors [21]. It has been deduced that cognitive factors such as speed of processing and verbal memory were more related to mood disturbances than level of physical independence with a contribution of $51.3 \%$ and $38.5 \%$ for both depression and anxiety, respectively [21].

In order to improve overall physical function among stroke survivors, emotional disturbances such as depressive symptoms require management because both cognition and mood impairments are associated $[21,22]$. Knowledge of the risk factors for cognitive impairments in stroke survivors may be useful in the holistic management. Thus, the aim of this review was to identify risk factors that include demographic, clinical, psychological, and physical determinants that may likely influence cognitive function among stroke survivors.

(1) Cognitive Impairment in Stroke Survivors. Cognitive impairment is a spectrum of intellectual decline with mild to severe cognitive functional deterioration [23]. Some of the researchers used the terms mild cognitive impairment (MCI) and dementia to represent cognitive impairment [2427]. MCI is a transitional phase between healthy aging and dementia. It involves impairment in memory domains that was reported by stroke survivors or their caregivers. Other cognitive domains normally remain intact and stroke survivors have the ability to perform activities of daily living (ADL). In contrast with MCI, dementia occurs where patients experience severe memory loss, impairments in other cognitive domains, and decline in physical function [24, 25]. Statistics showed that approximately $1-25 \%$ of older adults with MCI per year are at high risk to develop Alzheimer's disease $[28,29]$. Meanwhile, progression rate of MCI to dementia in 4 -year period is $12 \%$ per year in stroke survivors as compared to progression rate of normal adults to dementia with only $1-2 \%$ per year within 10 -year period [29]. This is a concern and calls for prevention of further deterioration as stroke will cause more severe cognitive damage.

In a study by Sachdev et al. [27], it was found that $58 \%$ of stroke survivors had cognitive impairment with a quarter of them diagnosed with dementia. It has also been reported that a stroke will double the risk of dementia [30]. In a Latin American study, 66\% and 61\% of stroke survivors were cognitively impaired at three and 12 months of the study $(39 \%$ cognitive impairment with no dementia; 22\% demented) [31]. The prevalence of cognitive impairment remains $21 \%$ at 3 months after stroke and after 14 years of follow-up period [32]. Prevalence varied due to differences in study population with nonlacunar and lacunar stroke being common in hospital and community based studies, respectively [33]. There is higher cognitive decline after lacunar stroke due to pathological causes where small vessel disease (SVD) affects wide region of the brain compared to nonlacunar stroke that involves extracranial region [33].

A cross-sectional study in United Kingdom (UK) found that the prevalence of cognitive impairment was high in the first month after ischemic stroke. This impairment involved speed and attention, frontal executive, nominal skills, perceptual skills, and visual memory [38]. Hurford et al. [38] reported that stroke survivors had improvement in visuospatial neglect and persistent impairment in speed and attention domain after 3 months of stroke. In a range of studies, researchers found that prevalence of cognitive impairment ranged from $7.5 \%$ to $72 \%$ from different studies which involved stroke survivors who had dementia and cognitive impairment with no dementia (CIND). The common terms for cognitive impairment that are used to refer to poststroke are cognitive impairment (PSCI), mild cognitive impairment (MCI), CIND, vascular cognitive impairment (VaCI), vascular mild cognitive impairment (VaMCI) subjective cognitive impairment (SCI), age-associated cognitive decline (AACD) dementia, vascular dementia $(\mathrm{VaD})$, poststroke dementia (PSD), and cognitive dysfunction.

\section{Methodology}

Searches were conducted using three databases (i.e., Medline, Scopus, and ISI Web of Science). Researchers used the same search terms for all databases: "stroke" OR "cerebrovascular disease" OR "cerebrovascular accident" OR "CVA" AND "mild cognitive impairment" OR "cognitive impairment" OR "MCI". There were 37 articles in Medline, 1654 articles in Scopus, and 240 articles in ISI Web of Science. Searches were refined by identifying the studies published in the years 2005-2015, full-text English articles, and adult aged as studied population. In addition, researcher restricted searching to area of study in psychology as an additional search criterion. Three hundred and twenty-six retrieved articles were screened using their titles and abstracts. Only 32 articles were related to review objectives and reviewed (12 articles in Medline, 7 articles in Scopus, and 13 articles in ISI Web of Science). Articles related to prestroke cognitive impairment, studies on validation of measurement tools, and molecular factors among stroke survivors were excluded from this review. Only 27 articles were included in this review after excluding five similar articles from different databases (Figure 1). Table 1 depicts the following features of related studies: (a) study reference, (b) objective of study, (c) sample size, (d) methodology, (e) setting, (f) prevalence of CI, and 


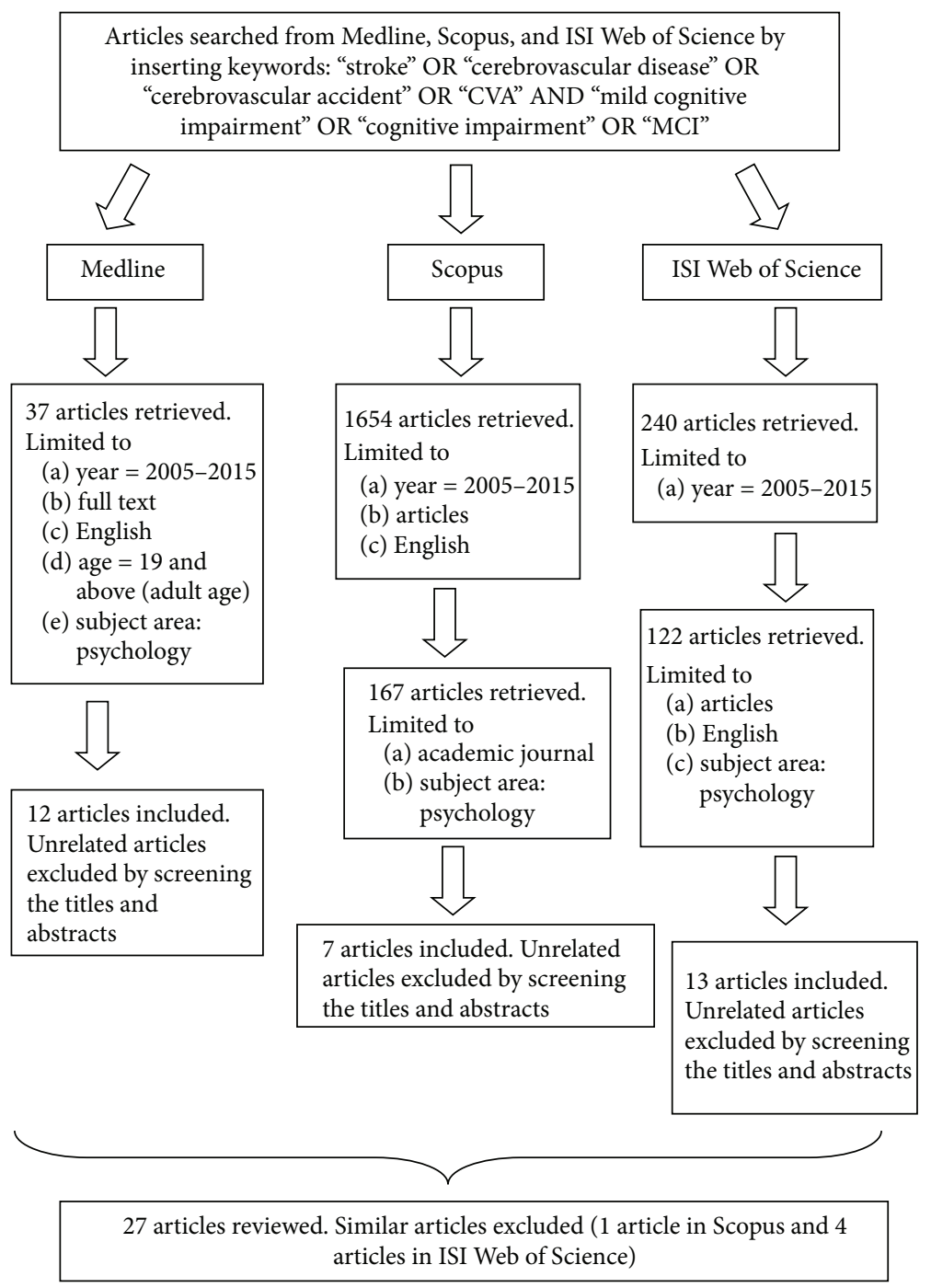

FIGURE 1: Flow chart of the reviewing process.

(d) outcomes (i.e., demographic, clinical, psychological, and physical).

\section{Results}

A total of 27 studies fulfilled this review's inclusion criteria in identifying demographic, clinical, psychological, and physical related risk factors that are likely to influence cognitive function among stroke survivors (refer to Table 1). Most of the studies (18 studies/67\%) were cross-sectional in manner $[17,27,34,37-39,41-50,54,55]$ and $74 \%$ (20 studies) were carried out in hospital settings [27, 35, 37-39, 41, 42, 44-46, 48-57]. Only three studies covered all the related risk factors in this review $[35,45,46]$, whereas other studies discussed some parts of it. Eighteen articles included demographic factors in the discussion mainly age, education level, sex, marital status, life styles, family history, and socioeconomic status $[17,27,32,34,35,37,40,42,44-46,49,51,52,54-$ 57]. Seventeen studies conferred the influence of clinical factors including medical, vascular, and neurological factors on cognitive impairment $[17,27,32,35,37,40,42,44-46$, $49,51,52,54-57]$. Depression was the main psychological factor of interest among researchers in 9 of the articles with psychiatric illnesses and stress as the most often mentioned, in spite of different psychological scales used in the studies [27, 35, 41, 44-46, 54, 55, 57]. Meanwhile, seven studies predicted the influence of physical functioning on cognitive impairment among stroke survivors [35, 36, 39-41, 45, 46].

3.1. Demographic Factors and Cognitive Impairment. One of the main predictors for cognitive impairment in stroke survivors reported in most studies was increased age $[17,27$, $32,34,35,37,40,45,49,51,52,54,57]$. Meanwhile, a lower level of education was highlighted as a risk factor for cognitive impairment in half of the studies $[17,34,37,42,45,46,49$, $50,54]$. Older stroke survivors with lower education level had higher prevalence of having cognitive impairment and history of stroke accelerated the development of dementia in them [34]. Life styles and dietary factors were also reported as risks for cognitive decline in stroke survivors. 


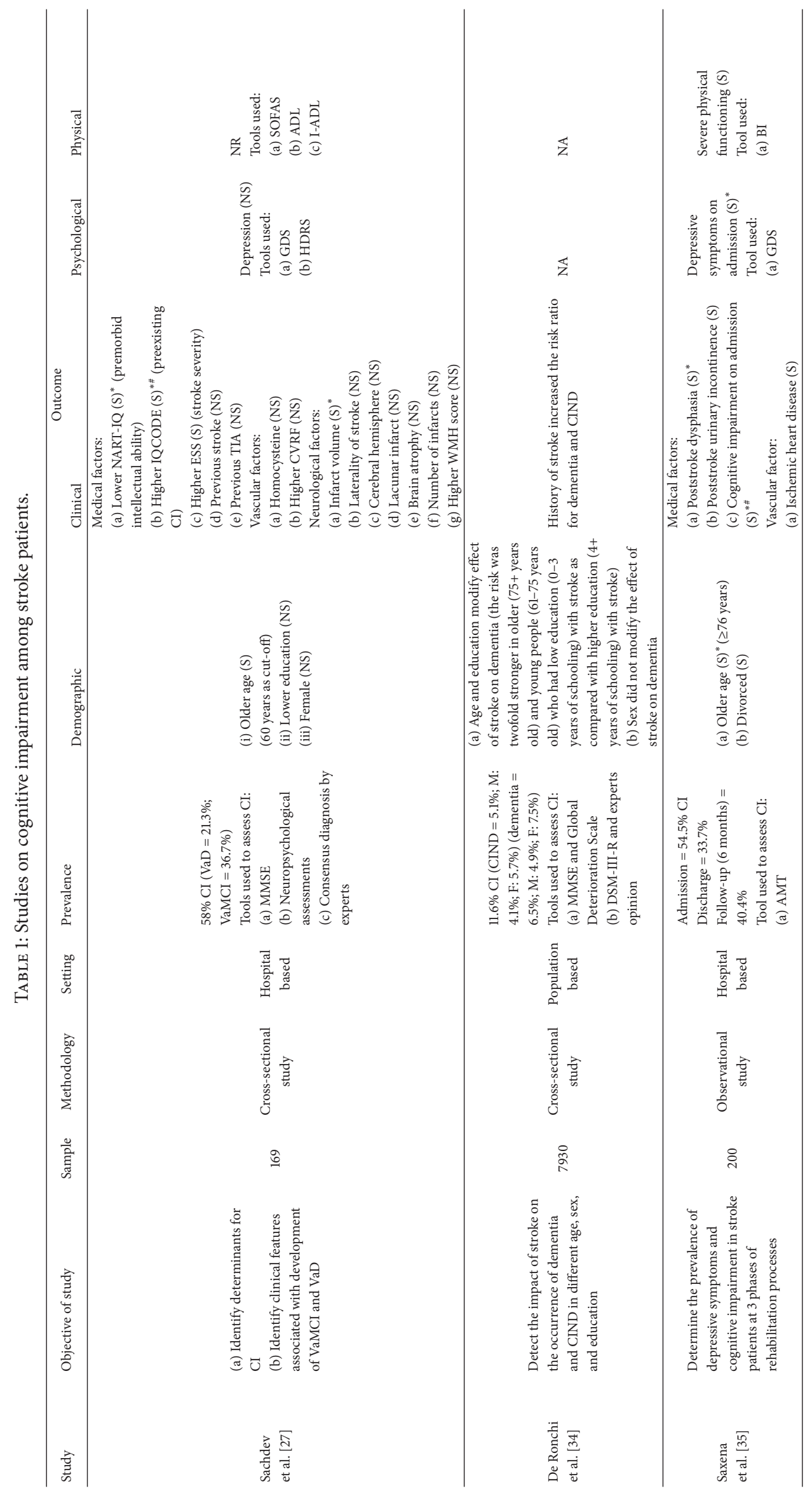




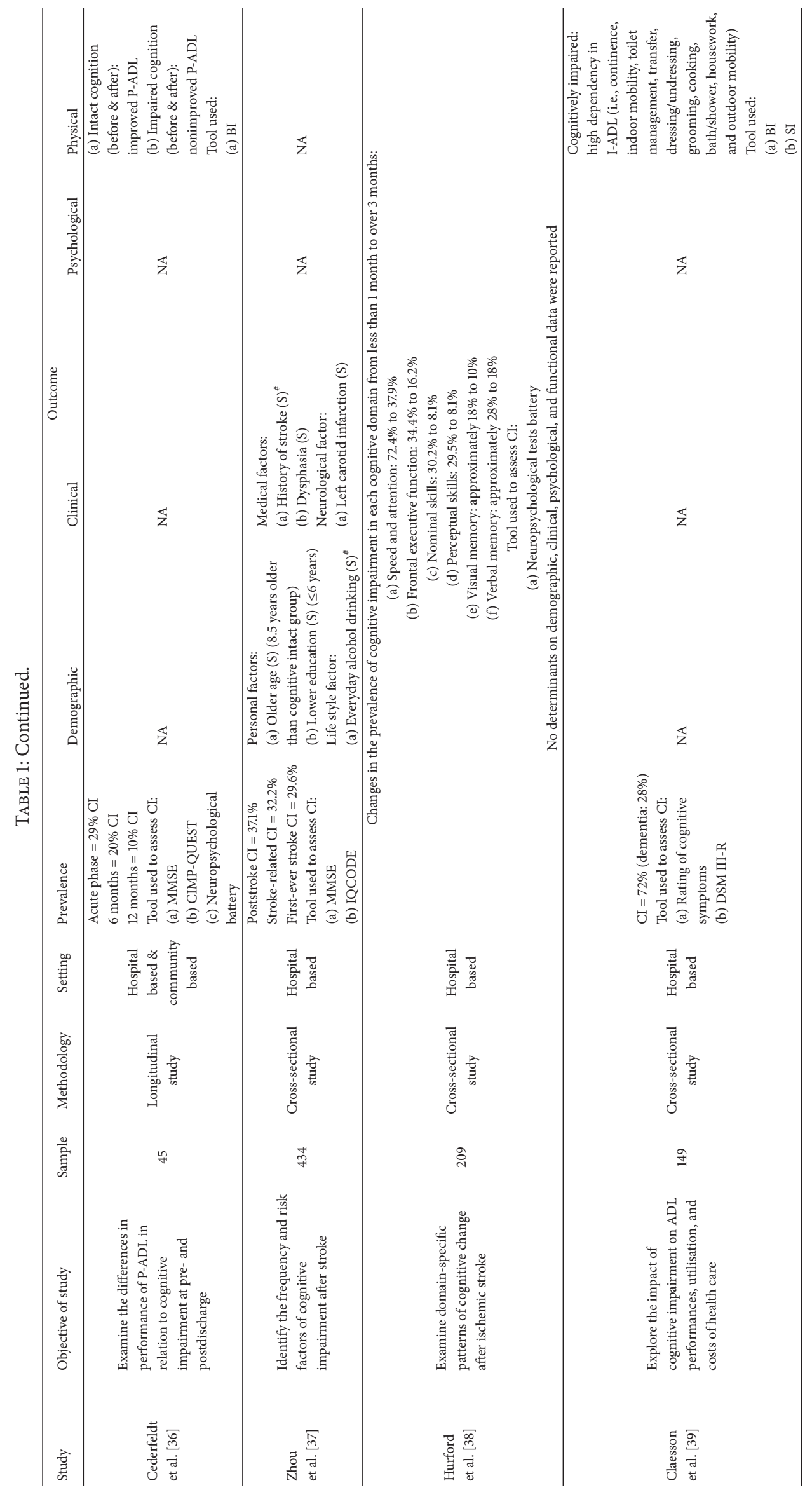




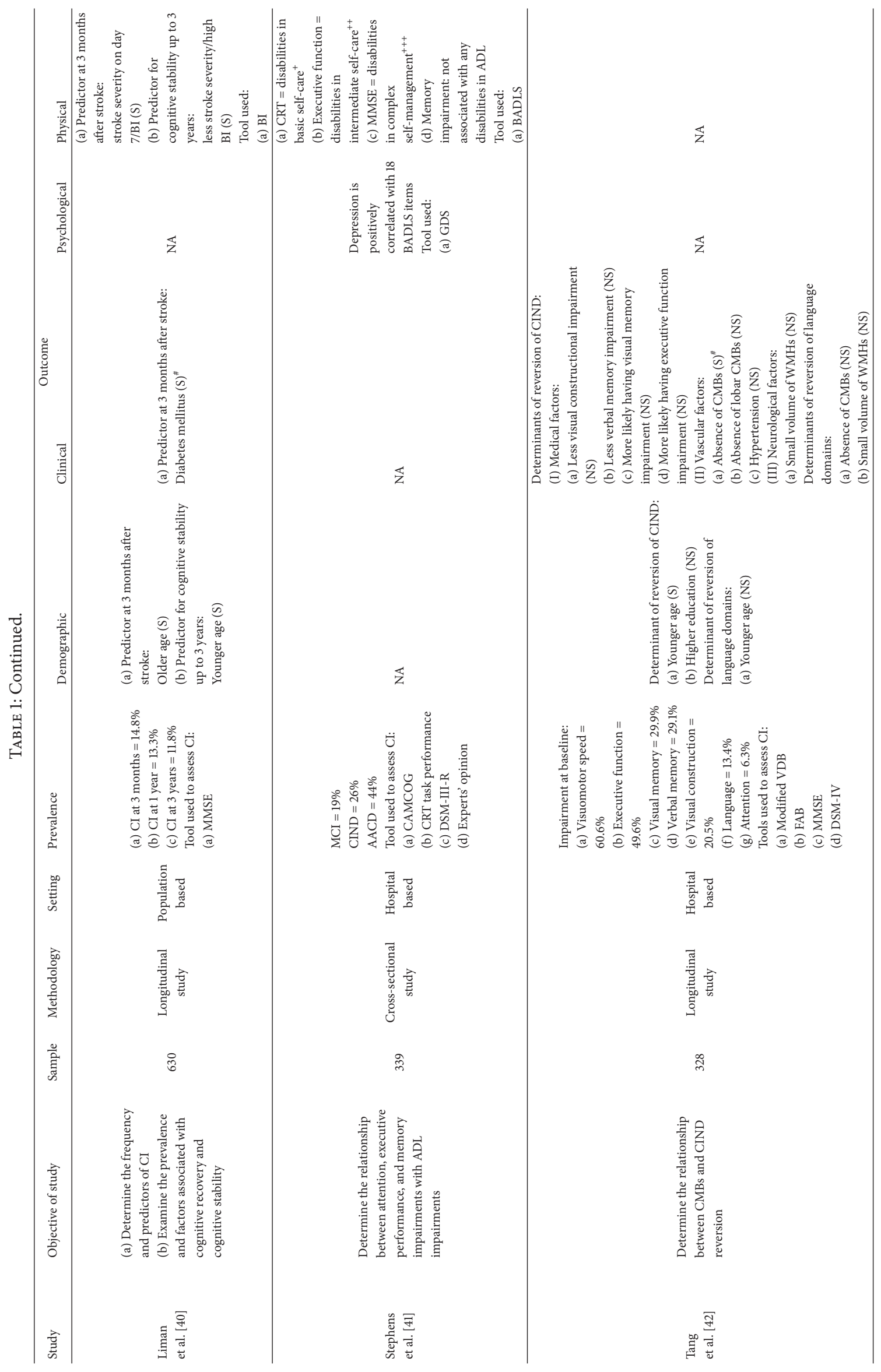




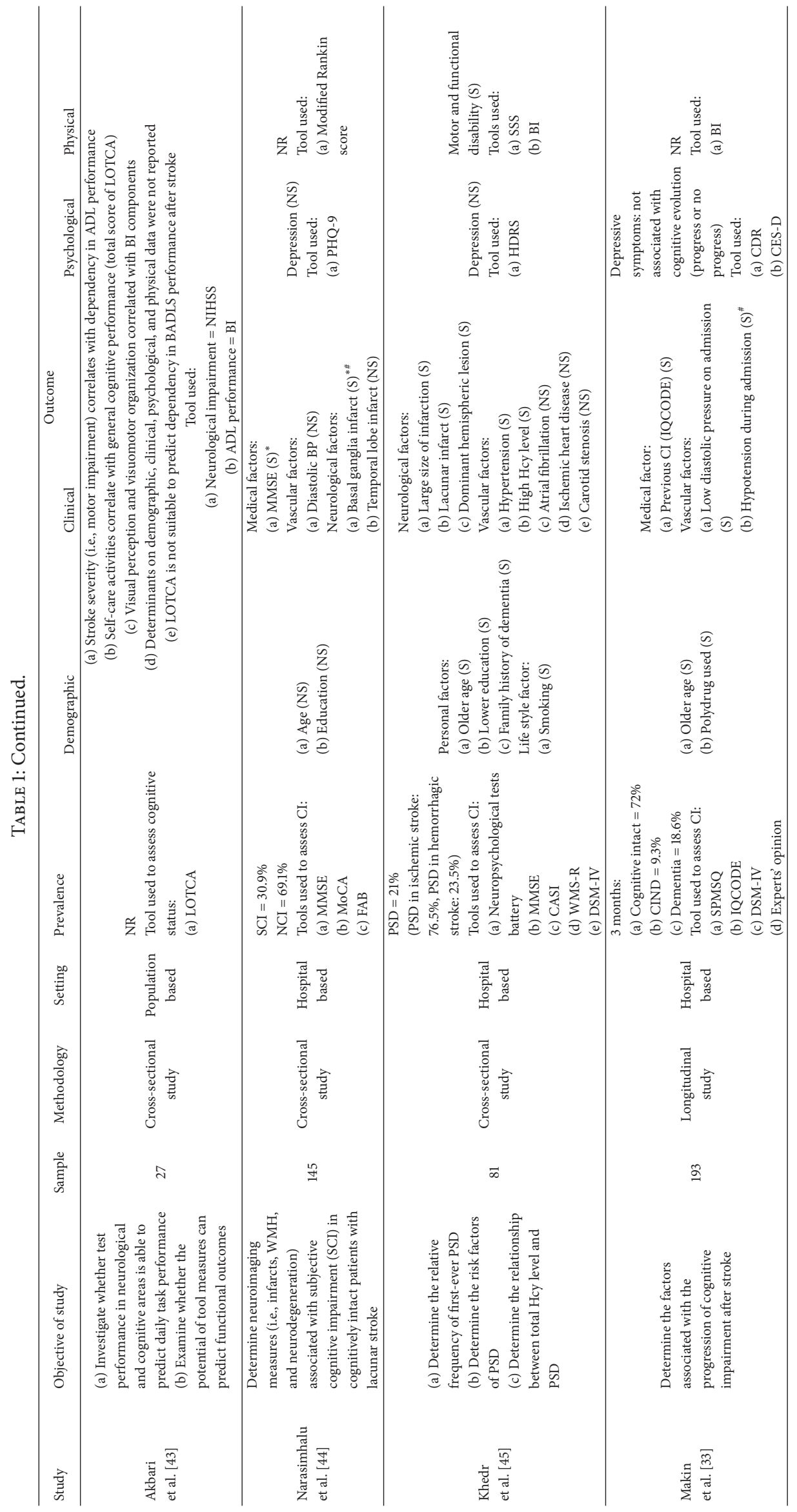




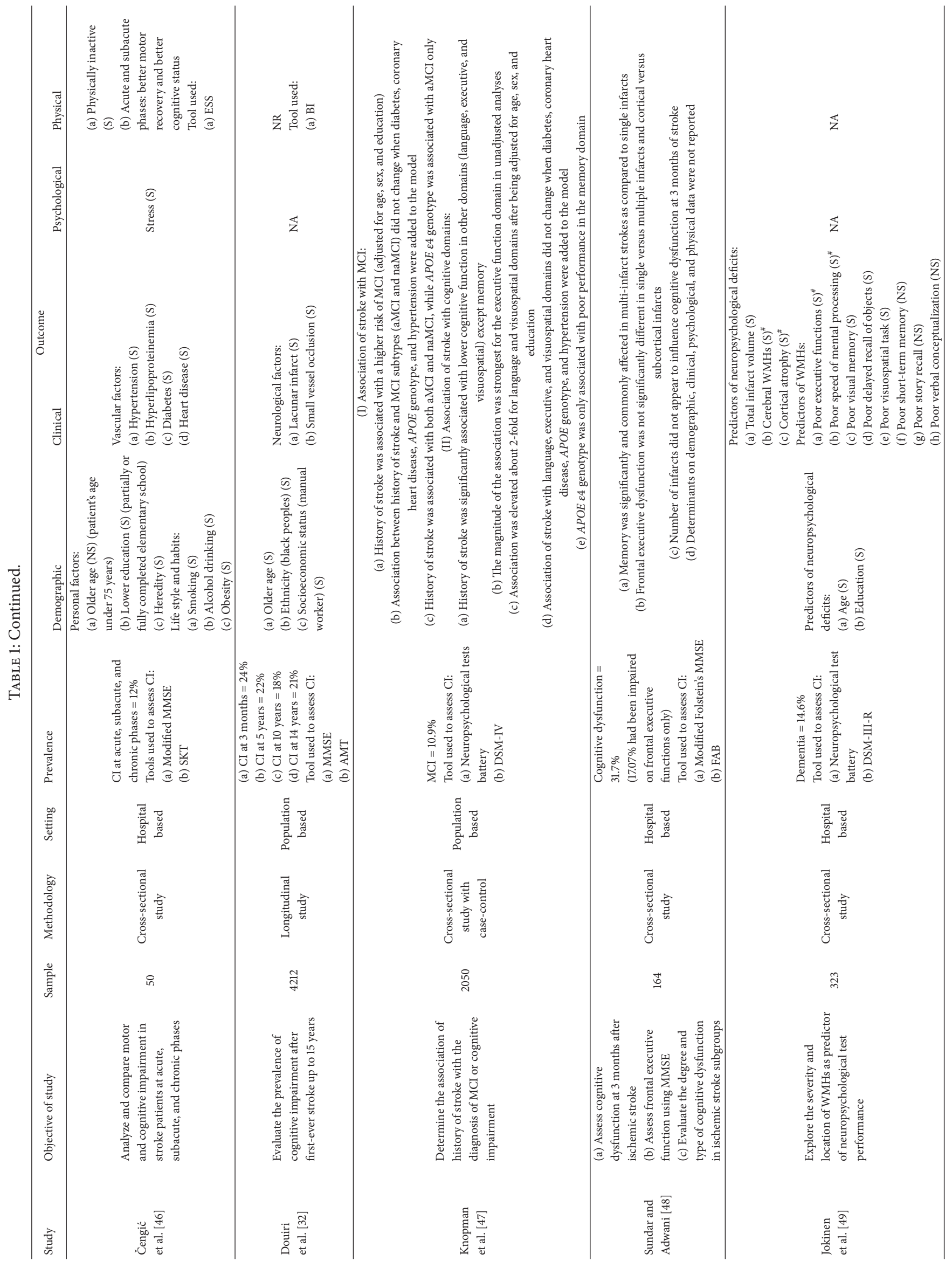




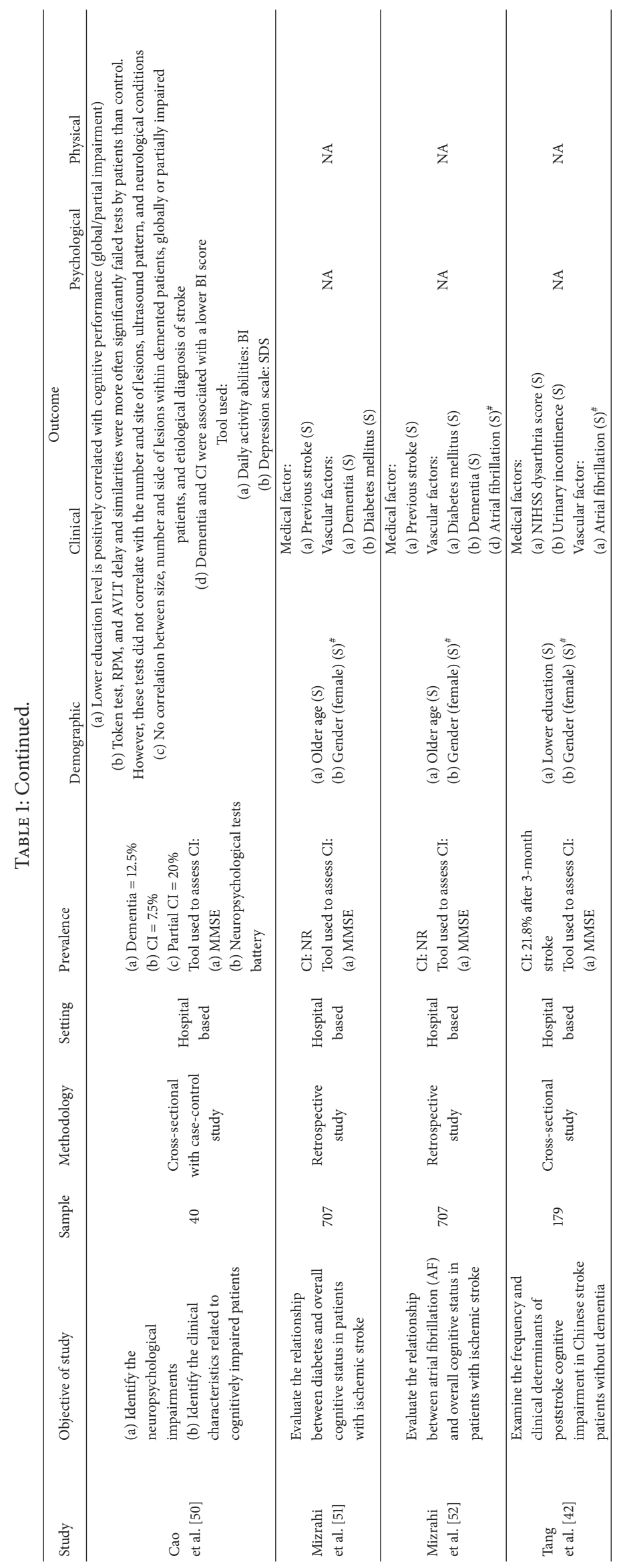




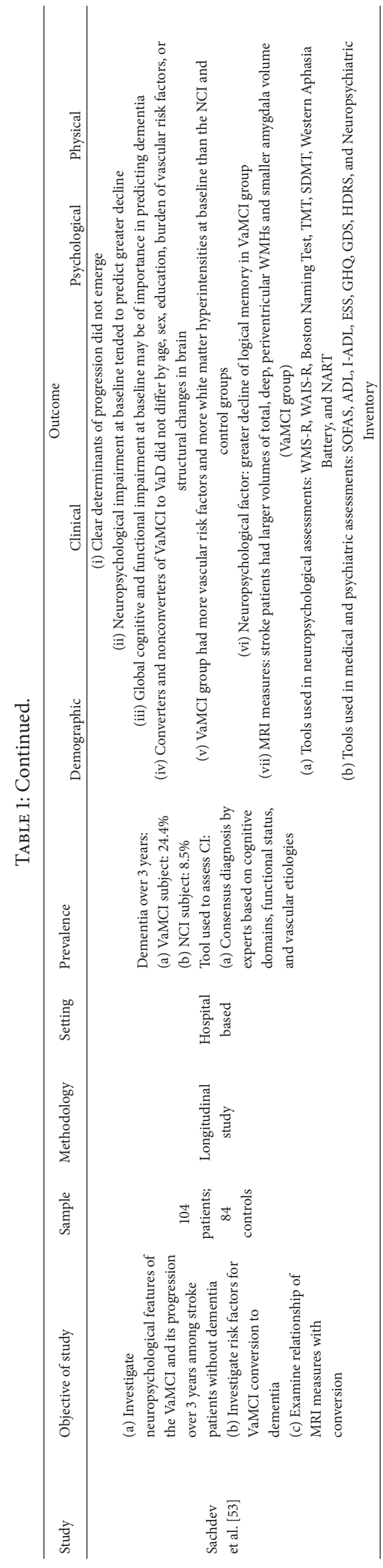

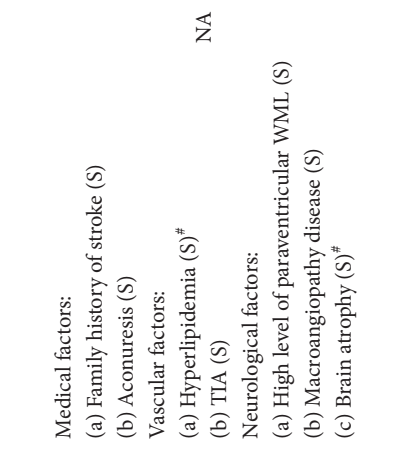
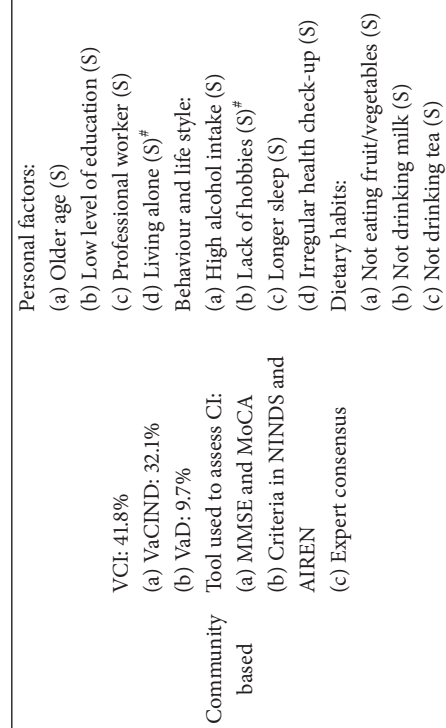

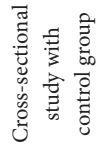

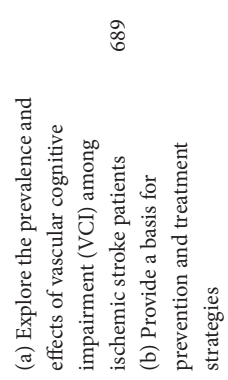

झ

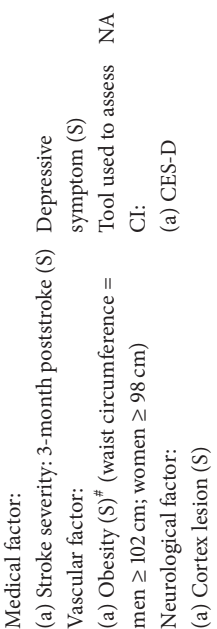

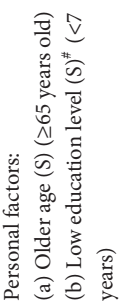

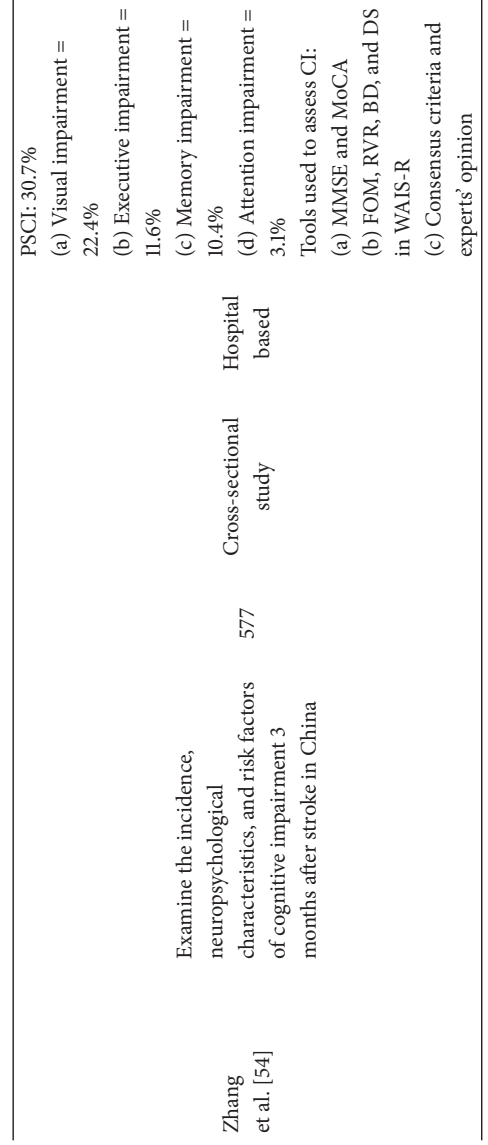




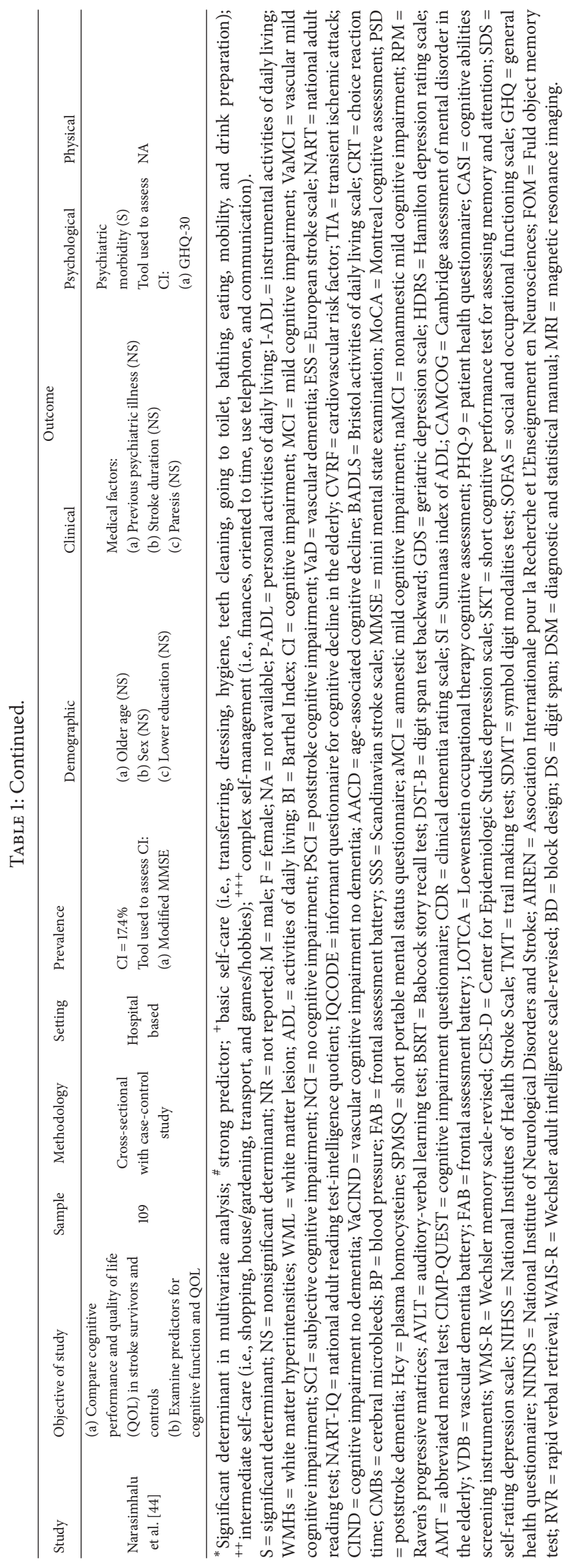


Four Asian studies conducted at hospital and community settings in China found that increased alcohol intake, lack of hobbies, longer sleep, irregular health check-up, and less vegetables, fruits, milk, and tea intake were identified as potential risk factors of poststroke cognitive impairment $[17,37,54,56]$.

Gender, family history of dementia, smoking habits, socioeconomic status, marital status, and ethnicity affected cognitive functioning $[17,27,32,34,35,42,45,46,51,52,55]$. In spite of the decline in cognitive status, approximately, $8 \%$ of stroke survivors improved from demented to nondemented state and $80 \%$ had stable cognitive functions after 24 -month period [57]. Stroke survivors who had progressed to dementia were noted to be older, had history of cognitive impairment, and were prescribed with more medications [57]. Cognitive impairment was also high among black compared to white ethnic group. Meanwhile, manual workers were at a higher risk in comparison to nonmanual workers (UK occupational codes) [32].

3.2. Clinical Factors and Cognitive Impairment. Studies showed that stroke survivors who had medical conditions, such as low premorbid intellectual ability, preexisting cognitive impairment, and high stroke severity, and history of stroke and transient ischemic stroke (TIA), poststroke dysphasia, and urinary incontinence (aconuresis) had higher risk of cognitive decline [17, 27, 34, 35, 37, 44, 51, 52, 54, 57]. Vascular factors contributed more than medical factors as the predictors were heart disease, diabetes mellitus, atrial fibrillation, hyperlipidemia, TIA, obesity, high homocysteine level, presence of cerebral microbleeds, low diastolic pressure, hypotension, dementia, hypertension, and hyperlipoproteinemia that appeared as significant determinants in multivariate analysis [17, 30, 35, 45, 46, 51, 52, 54, 56, 57].

Neuroimaging characteristics which explained neurological factors of stroke were also the most influential determinants for poststroke cognitive impairment. There were nine studies reporting on neurological factors. Researchers indicated that infarct volume, left carotid infarction, high level of paraventricular white matter lesion (WML), brain atrophy, basal ganglia infarct, large infarct volume, more cerebral white matter hyperintensities (WMHs), cortical atrophy, lacunar infarct, small vessel occlusion, and dominant hemispheric lesions and cortex lesion were the significant risk factors for cognitive impairment [27, 32, 37, 44, 45, 49, 54]. On the other hand, two studies that provided neuroimaging information deduced that area of infarction (i.e., cortical, subcortical), type of infarctions (i.e., single, multiple), and size of lesion were found not to be correlated with frontal executive dysfunction and cognitive status $[48,50]$. A study by Sachdev et al. [27] showed that stroke survivors who were in VaMCI group had larger volume of total, deep, and periventricular WMHs. It was also found that VaMCI group had smaller amygdala volume than noncognitive impaired (NCI) and control groups [27]. Besides that, a few researchers studied biomarkers as potentials to predict cognitive functional status. The authors showed that blood plasma homocysteine, APOE $\varepsilon 4$ genotype, HbAlc, LDL, and HDL were clinical markers for cognitive status [44, 45, 47].
3.3. Psychological Distress and Cognitive Impairment. The common psychological distress among stroke survivors regardless of the severity of cognitive impairment was depression $[35,45,47]$. Depressive symptoms were reduced over time from time of admission to 6 months after stroke in nearly half of the stroke survivors with decrement in cognitive impairment [35]. In contrast, del Ser et al. [57] reported that depression does not associate with cognitive decline at 3month period after stroke. It was found that four studies showed depression and psychological problems had some significant effects as risk factors for cognitive impairment $[35,46,54,55]$. Inconsistent findings were reported in other studies [27, 44, 45, 57].

3.4. Physical Function and Cognitive Impairment. Stroke survivors who are physically dependent and more impaired usually perform poorly in tasks which require higher-order cognitive functions such as motor control, organization, problem solving, and memory. Lower performances in cognitive functions were positively associated with dependency in activity of daily living (ADL) [58]. It is a main concern after stroke because impairment in information processing is related to control of motor movement in executing ADL [5961] Those who had intact prestroke cognition were reported to have higher personal ADL (P-ADL) and Barthel Index (BI) score compared to those who had cognitive impairment [36]. Stroke survivors with impaired cognitive impairment are highly dependent on instrumental ADL (I-ADL) which requires more complex tasks such as cooking, housework, and outdoor mobility in comparison to personal activities (i.e., eating, continence, bath, and dressing) [39].

Meanwhile, Liman et al. [40] reported differently where stroke severity was measured using Barthel Index score and was a significant predictor of cognitive impairment after 3 months of stroke. Stephens et al. [41] found that different impairment in cognitive domains would affect physical abilities differently. Researchers explained that those who had impairment in (1) reaction time would face disabilities in basic self-care, (2) impairment in executive function is associated with disabilities in intermediate self-care, (3) impairment in MMSE is associated with complex selfmanagement, and (4) deficit in memory is associated with any basic, intermediate, and complex ADL components. Selfcare activities were the main outcome measures examined because self-care activities correlate with general cognitive performance such as visual perception and visuomotor organization [43]. Other studies also found that stroke severity and lower Barthel Index score were significant predictors for poststroke dementia and other types of cognitive impairment $[35,45,50]$.

\section{Discussion}

Findings from the current review suggested that there were various risk factors of cognitive impairment among stroke survivors. In terms of sociodemographic factors, age and education level were found to be the main predictors of cognitive impairment. Older adults were severely impaired in cognition due to the nature of stroke that accelerates 
cognitive decline [34]. It is deduced that stroke survivors with higher education level were less likely to be affected in their cognitive functioning because they have larger brain reserve capacity which can compensate for the brain damage [62].

Clinical factors were also main risk factors of cognitive impairment in stroke survivors. History of hypertension and diabetes may reduce the brain volume and also cause white matter lesions [63]. Affected regions of brain and types of stroke were also important variables which determined cognitive status of stroke survivors because lacunar infarcts and cortical strokes (i.e., parietal, cingulated, premotor, occipital, and temporal cortex) caused more severe deficits in cognitive status $[64,65]$. More severe outcomes were noted when the stroke occurred at the important regions of brain which controlled vital functions.

In a study on the effects of blood pressure, low diastolic blood pressure and episode of hypotension were significantly associated with cognitive declines [57]. In contrast, a study conducted among MCI patient with high blood pressure showed that stroke survivors' performance deteriorated in tasks requiring rapid responses and set shifting (TMT A and B) and in expressive language (naming test) [66]. Therefore, it is questionable if hypertension leads to hemorrhagic stroke and vascular cognitive impairment. In addition, vascular cognitive impairment occurs due to impairment in bloodbrain barrier and white matter lesion and increase in vascular permeability due to endothelial cell retraction which may also involve the blood flow [67]. Cerebral microbleeds in stroke survivors with lacunar infarcts had significant correlation with cognitive impairment. According to Zhang et al. [68] thalamic microbleeds reduced the orientation subscore, lobar (cortex/subcortex) microbleeds caused low visuospatial/executive subscores, and basal ganglia microbleeds were found to significantly reduce attention and visuospatial/executive subscores. The damage not only appeared at the affected area but also may disrupt other tissues nearby possibly due to lack of blood supply. Thus, pathological causes of infarction and tissue necrosis may have significant effect on cognitive function at the affected region [69].

Stroke had a significant impact on psychological wellbeing of stroke survivors. Stroke survivors usually experience sleep disturbances, low motivation, low self-esteem, and worries about their future due to restrictions and disabilities [70]. These psychological changes and stressful situations lead to depression and anxiety symptoms which affect their performance in executive function, memory, speed, and motor processing [71]. In addition, older adults who were highly anxious presented with cognitive decline and Alzheimer's disease after a year of cognitive impairment [72].

Mood changes and chronic stress due to physical limitations, restrictions in $\mathrm{ADL}$, and low motivation were possible factors that caused emotional distress among stroke survivors [73]. Depression was believed to reduce the effort in cognitive test in older adults and resulted in poorer performance in the test [58]. This is due to declined cognitive processing which is related to slowness in responses and having difficulties to keep track in conversation. On the other hand, depression, somatisation, and insufficiency of thinking and acting were the most frequent psychiatric symptoms which were reported to have problem in executive and global cognitive function (i.e., orientation, attention, praxis, and language) [20]. Severe cases of cognitive impairment such as vascular dementia were also reported to present with more psychiatric symptoms than the milder ones [20].

Poor performance in executive function can affect individual's ADL and increase their risk for poststroke disability [74]. In addition, cognitive impairment also disrupts their orientation, perception, thinking process, and memory leading to dependence in self-care activities [43]. Stroke survivors with higher functional impairment were likely to be diagnosed with cognitive impairment with no dementia (CIND) [35].

There were two factors which caused cognitively impaired stroke survivors to have poor functional outcomes and these include higher stroke severity and poorer compliance to rehabilitation [75]. Assessment of cognitive status at initial stage of stroke is very important to determine functional outcomes. Physical performance of stroke survivors after 6 months of stroke was associated with cognitive impairment on admission and also cognitive improvement over 6 months [22]. This indicates that physical function was independently associated with cognitive status which was the main variable affected after stroke.

Identifying poststroke risk factors for cognitive impairment at early stage is important. Even more important is to evaluate the contribution of each risk factor towards cognitive impairment. Furthermore, current existing literature is lacking on integrating risk factors which increases poststroke risk for cognitive impairment. Previous studies mainly focus on identifying and grouping risk factors (e.g., $[45,76,77])$ rather than providing reliable information or data in predicting and reducing the risk for poststroke cognitive impairment. Future studies should focus on developing research on identifying and enhancing protecting factors against poststroke cognitive impairment. In addition, researchers need to explore combined effect of both pharmacotherapy and nonpharmacotherapy, especially psychosocial intervention to improve poststroke cognitive function. There is also a need for future research to focus on clinical and evaluative studies on holistic intervention to establish clinical effect size, especially on modifiable risk factors. The impact of modifiable risk factors on nonmodifiable risk factors to develop cognitive impairment needs more systematic research approach.

\section{Conclusion}

This review indicated that age, level of education, history of stroke, diabetes mellitus, hypertension, types of stroke, affected region, size and location of infarction, depressive symptoms, and physical function were the potential factors that determine cognitive status of stroke survivors. Identifying these risk factors would be beneficial for clinician and healthcare practitioners in the management of stroke survivors. This will assist in the prevention of further cognitive decline and improve psychological wellbeing through effective intervention. Further research examining in-depth and multiple risk factors discussed in this review is warranted. 


\section{Disclosure}

This review is a part of fulfilment of master thesis project that was conducted in a community based study.

\section{Competing Interests}

The authors declare that there is no conflict of interests regarding the publication of this paper.

\section{Acknowledgments}

Researchers would like to express their gratitude to the National Stroke Association of Malaysia (NASAM) and Pusat Pemulihan Kesihatan-PERKIM for their help and support in this study.

\section{References}

[1] American Heart Association, Heart Disease and Stroke Statistics-2003 Update, American Heart Association, Dallas, Tex, USA, 2002.

[2] M. Hommel, S. T. Miguel, B. Naegele, N. Gonnet, and A. Jaillard, "Cognitive determinants of social functioning after a first ever mild to moderate stroke at vocational age," Journal of Neurology, Neurosurgery and Psychiatry, vol. 80, no. 8, pp. 876-880, 2009.

[3] J. W. Sturm, "Handicap after stroke: how does it relate to disability, perception of recovery, and stroke subtype? The North East Melbourne Stroke Incidence Study (NEMESIS)," Stroke, vol. 33, no. 3, pp. 762-768, 2002.

[4] C. D. A. Wolfe, S. L. Crichton, P. U. Heuschmann et al., "Estimates of outcomes up to ten years after stroke: analysis from the prospective South London stroke register," PLoS Medicine, vol. 8, no. 5, Article ID e1001033, 2011.

[5] J. R. Hodges, Cognitive Assessment for Clinicians, Oxford University Press, Oxford, UK, 2011.

[6] T. K. Tatemichi, D. W. Desmond, Y. Stern, M. Paik, M. Sano, and E. Bagiella, "Cognitive impairment after stroke: frequency, patterns, and relationship to functional abilities," Journal of Neurology Neurosurgery and Psychiatry, vol. 57, no. 2, pp. 202207, 1994

[7] J. H. Kramer, B. R. Reed, D. Mungas, M. W. Weiner, and H. C. Chui, "Executive dysfunction in subcortical ischaemic vascular disease," Journal of Neurology, Neurosurgery \& Psychiatry, vol. 72, no. 2, pp. 217-220, 2002.

[8] J. S. Meyer, G. Xu, J. Thornby, M. H. Chowdhury, and M. Quach, "Is mild cognitive impairment prodromal for vascular dementia like Alzheimer's disease?” Stroke, vol. 33, no. 8, pp. 1981-1985, 2002.

[9] P. Appelros, I. Nydevik, and A. Terént, "Living setting and utilisation of ADL assistance one year after a stroke with special reference to gender differences," Disability and Rehabilitation, vol. 28, no. 1, pp. 43-49, 2006.

[10] S. G. Parker, R. Fadayevatan, and S. D. Lee, "Acute hospital care for frail older people," Age and Ageing, vol. 35, no. 6, pp. 551-552, 2006.

[11] P. J. Clarke, V. Blount, and A. Colantonio, "Cognitive impairment predicts fatal incident stroke: findings from a national sample of older adults," Journal of the American Geriatrics Society, vol. 59, no. 8, pp. 1490-1496, 2011.
[12] M. Danovska and D. Peychinska, "Post-stroke cognitive impairment-phenomenology and prognostic factors," Journal of IMAB-Annual Proceeding (Scientific Papers), vol. 8, no. 3, pp. 290-297, 2012.

[13] J.-H. Sun, L. Tan, and J.-T. Yu, "Post-stroke cognitive impairment: epidemiology, mechanisms and management," Annals of Translational Medicine, vol. 2, no. 8, p. 80, 2014.

[14] R. H. Swartz, M. Bayley, K. L. Lanctot et al., "Post-stroke depression, obstructive sleep apnea, and cognitive impairment: rationale for, and barriers to, routine screening," International Journal of Stroke, 2016.

[15] D. L. Coco, G. Lopez, and S. Corrao, "Cognitive impairment and stroke in elderly patients," Vascular Health and Risk Management, vol. 12, pp. 105-116, 2016.

[16] B. Ovbiagele, J. L. Saver, N. Sanossian et al., "Predictors of cerebral microbleeds in acute ischemic stroke and TIA patients," Cerebrovascular Diseases, vol. 22, no. 5-6, pp. 378-383, 2006.

[17] Q. Tu, B. Ding, X. Yang et al., "The current situation on vascular cognitive impairment after ischemic stroke in Changsha," Archives of Gerontology and Geriatrics, vol. 58, no. 2, pp. 236247, 2014.

[18] A. Verdelho, S. Madureira, J. M. Ferro et al., "Physical activity prevents progression for cognitive impairment and vascular dementia: results from the LADIS (Leukoaraiosis and Disability) study," Stroke, vol. 43, no. 12, pp. 3331-3335, 2012.

[19] M. Ihara, Y. Okamoto, Y. Hase, and R. Takahashi, "Association of physical activity with the visuospatial/executive functions of the montreal cognitive assessment in patients with vascular cognitive impairment," Journal of Stroke and Cerebrovascular Diseases, vol. 22, no. 7, pp. e146-e151, 2013.

[20] S. Rasquin, J. Lodder, and F. Verhey, "The association between psychiatric and cognitive symptoms after stroke: a prospective study," Cerebrovascular Diseases, vol. 19, no. 5, pp. 309-316, 2005.

[21] S. L. Barker-Collo, "Depression and anxiety 3 months poststroke: prevalence and correlates," Archives of Clinical Neuropsychology, vol. 22, no. 4, pp. 519-531, 2007.

[22] S. K. Saxena, T.-P. Ng, G. Koh, D. Yong, and N. P. Fong, "Is improvement in impaired cognition and depressive symptoms in post-stroke patients associated with recovery in activities of daily living?" Acta Neurologica Scandinavica, vol. 115, no. 5, pp. 339-346, 2007.

[23] T. K. Tatemichi, D. W. Desmond, Y. Stern, M. Paik, M. Sano, and E. Bagiella, "Cognitive impairment after stroke: frequency, patterns, and relationship to functional abilities," Journal of Neurology, Neurosurgery, and Psychiatry, vol. 57, no. 2, pp. 202207, 1994.

[24] A. Busse, M. C. Angermeyer, and S. G. Riedel-Heller, "Progression of mild cognitive impairment to dementia: a challenge to current thinking," The British Journal of Psychiatry, vol. 189, pp. 399-404, 2006.

[25] H. Chertkow, F. Massoud, Z. Nasreddine et al., "Diagnosis and treatment of dementia: 3. Mild cognitive impairment and cognitive impairment without dementia," Canadian Medical Association Journal, vol. 178, no. 10, pp. 1273-1285, 2008.

[26] J. C. Morris, M. Storandt, J. P. Miller et al., "Mild cognitive impairment represents early-stage Alzheimer disease," Archives of Neurology, vol. 58, no. 3, pp. 397-405, 2001.

[27] P. S. Sachdev, H. Brodaty, M. J. Valenzuela et al., "Clinical determinants of dementia and mild cognitive impairment following ischaemic stroke: the Sydney Stroke Study," Dementia 
and Geriatric Cognitive Disorders, vol. 21, no. 5-6, pp. 275-283, 2006.

[28] B. Dawe, A. Procter, and M. Philpot, "Concepts of mild memory impairment in the elderly and their relationship to dementia-a review," International Journal of Geriatric Psychiatry, vol. 7, no. 7, pp. 473-479, 1992.

[29] R. C. Petersen, G. E. Smith, S. C. Waring, R. J. Ivnik, E. G. Tangalos, and E. Kokmen, "Mild cognitive impairment: clinical characterization and outcome," Archives of Neurology, vol. 56, no. 3, pp. 303-308, 1999.

[30] D. Leys, H. Hénon, M.-A. Mackowiak-Cordoliani, and F. Pasquier, "Poststroke dementia," The Lancet Neurology, vol. 4, no. 11, pp. 752-759, 2005.

[31] C. Delgado, A. Donoso, P. Orellana, C. Vásquez, V. Díaz, and M. I. Behrens, "Frequency and determinants of poststroke cognitive impairment at three and twelve months in Chile," Dementia and Geriatric Cognitive Disorders, vol. 29, no. 5, pp. 397-405, 2010.

[32] A. Douiri, A. G. Rudd, and C. D. A. Wolfe, "Prevalence of poststroke cognitive impairment: South London stroke register 1995-2010," Stroke, vol. 44, no. 1, pp. 138-145, 2013.

[33] S. D. J. Makin, S. Turpin, M. S. Dennis, and J. M. Wardlaw, "Cognitive impairment after lacunar stroke: systematic review and meta-analysis of incidence, prevalence and comparison with other stroke subtypes," Journal of Neurology, Neurosurgery \& Psychiatry, vol. 84, no. 8, pp. 893-900, 2013.

[34] D. De Ronchi, K. Palmer, P. Pioggiosi et al., "The combined effect of age, education, and stroke on dementia and cognitive impairment no dementia in the elderly," Dementia and Geriatric Cognitive Disorders, vol. 24, no. 4, pp. 266-273, 2007.

[35] S. K. Saxena, T. P. Ng, D. Yong, N. P. Fong, and G. Koh, "Subthreshold depression and cognitive impairment but not demented in stroke patients during their rehabilitation," Acta Neurologica Scandinavica, vol. 117, no. 2, pp. 133-140, 2008.

[36] M. Cederfeldt, G. Gosman-Hedström, C. G. Pérez, M. Sävborg, and E. Tarkowski, "Recovery in personal care related to cognitive impairment before and after stroke-a 1-year follow-up," Acta Neurologica Scandinavica, vol. 122, no. 6, pp. 430-437, 2010.

[37] D. H. D. Zhou, J. Y. J. Wang, J. Li, J. Deng, C. Gao, and M. Chen, "Frequency and risk factors of vascular cognitive impairment three months after ischemic stroke in China: the Chongqing stroke study," Neuroepidemiology, vol. 24, no. 1-2, pp. 87-95, 2005.

[38] R. Hurford, A. Charidimou, Z. Fox, L. Cipolotti, and D. J. Werring, "Domain-specific trends in cognitive impairment after acute ischaemic stroke," Journal of Neurology, vol. 260, no. 1, pp. 237-241, 2013.

[39] L. Claesson, T. Lindén, I. Skoog, and C. Blomstrand, "Cognitive impairment after stroke-impact on activities of daily living and costs of care for elderly people: the Göteborg 70+ stroke study," Cerebrovascular Diseases, vol. 19, no. 2, pp. 102-109, 2005.

[40] T. G. Liman, P. U. Heuschmann, M. Endres, A. Flöel, S. Schwab, and P. L. Kolominsky-Rabas, "Changes in cognitive function over 3 years after first-ever stroke and predictors of cognitive impairment and long-term cognitive stability: the Erlangen Stroke Project," Dementia and Geriatric Cognitive Disorders, vol. 31, no. 4, pp. 291-299, 2011.

[41] S. Stephens, R. A. Kenny, E. Rowan et al., "Association between mild vascular cognitive impairment and impaired activities of daily living in older stroke survivors without dementia," Journal of the American Geriatrics Society, vol. 53, no. 1, pp. 103-107, 2005.
[42] W. K. Tang, S. S. M. Chan, H. F. K. Chiu et al., "Frequency and clinical determinants of poststroke cognitive impairment in nondemented stroke patients," Journal of Geriatric Psychiatry and Neurology, vol. 19, no. 2, pp. 65-71, 2006.

[43] S. Akbari, H. Ashayeri, M. A. Fahimi, M. Kamali, and P. D. Lyden, "The correlation of independency in activities of daily living performance with cognitive status and the intensity of neurological impairment in right-handed stroke patients," NeuroRehabilitation, vol. 29, no. 3, pp. 311-316, 2011.

[44] K. Narasimhalu, L. Wiryasaputra, Y.-Y. Sitoh, and N. Kandiah, "Post-stroke subjective cognitive impairment is associated with acute lacunar infarcts in the basal ganglia," European Journal of Neurology, vol. 20, no. 3, pp. 547-551, 2013.

[45] E. M. Khedr, S. A. Hamed, H. K. El-Shereef et al., "Cognitive impairment after cerebrovascular stroke: relationship to vascular risk factors," Neuropsychiatric Disease and Treatment, vol. 5, no. 1, pp. 103-116, 2009.

[46] L. Čengić, V. Vuletić, M. Karlić, M. Dikanović, and V. Demarin, "Motor and cognitive impairment after stroke," Acta Clinica Croatica, vol. 50, no. 4, pp. 463-467, 2011.

[47] D. S. Knopman, R. O. Roberts, Y. E. Geda et al., "Association of prior stroke with cognitive function and cognitive impairment: a population-based study," Archives of Neurology, vol. 66, no. 5, pp. 614-619, 2009.

[48] U. Sundar and S. Adwani, "Post-stroke cognitive impairment at 3 months," Annals of Indian Academy of Neurology, vol. 13, no. 1, pp. 42-46, 2010.

[49] H. Jokinen, H. Kalska, R. Mäntylä et al., "White matter hyperintensities as a predictor of neuropsychological deficits poststroke," Journal of Neurology, Neurosurgery and Psychiatry, vol. 76, no. 9, pp. 1229-1233, 2005.

[50] M. Cao, M. Ferrari, R. Patella, C. Marra, and M. Rasura, "Neuropsychological findings in young-adult stroke patients," Archives of Clinical Neuropsychology, vol. 22, no. 2, pp. 133-142, 2007.

[51] E. H. Mizrahi, A. Waitzman, T. Blumstein, M. Arad, and A. Adunsky, "Diabetes mellitus predicts cognitive impairment in patients with ischemic stroke," American Journal of Alzheimer's Disease and other Dementias, vol. 25, no. 4, pp. 362-366, 2010.

[52] E. H. Mizrahi, A. Waitzman, M. Arad, and A. Adunsky, "Atrial fibrillation predicts cognitive impairment in patients with ischemic stroke," American Journal of Alzheimer's Disease and other Dementias, vol. 26, no. 8, pp. 623-626, 2011.

[53] P. S. Sachdev, X. Chen, H. Brodaty, C. Thompson, A. Altendorf, and W. Wen, "The determinants and longitudinal course of post-stroke mild cognitive impairment," Journal of the International Neuropsychological Society, vol. 15, no. 6, pp. 915-923, 2009.

[54] Y. Zhang, Z. B. Zhang, Y. Yang et al., "Incidence and risk factors of cognitive impairment 3 months after first-ever stroke: a cross-sectional study of 5 geographic areas of China," Journal of Huazhong University of Science and Technology, vol. 32, no. 6, pp. 906-911, 2012.

[55] F. O. Fatoye, M. A. Komolafe, B. A. Eegunranti, A. O. Adewuya, S. K. Mosaku, and G. K. Fatoye, "Cognitive impairment and quality of life among stroke survivors in Nigeria," Psychological Reports, vol. 100, no. 3, pp. 876-882, 2007.

[56] W. K. Tang, Y.-K. Chen, J.-Y. Lu et al., "Absence of cerebral microbleeds predicts reversion of vascular 'cognitive impairment no dementia' in stroke," International Journal of Stroke, vol. 6, no. 6, pp. 498-505, 2011. 
[57] T. del Ser, R. Barba, M. M. Morin et al., "Evolution of cognitive impairment after stroke and risk factors for delayed progression," Stroke, vol. 36, no. 12, pp. 2670-2675, 2005.

[58] J. Chodosh, D. Miller-Martinez, C. S. Aneshensel, R. G. Wight, and A. S. Karlamangla, "Depressive symptoms, chronic diseases, and physical disabilities as predictors of cognitive functioning trajectories in older Americans," Journal of the American Geriatrics Society, vol. 58, no. 12, pp. 2350-2357, 2010.

[59] N. Dancause, A. Ptito, and M. F. Levin, "Error correction strategies for motor behavior after unilateral brain damage: short-term motor learning processes," Neuropsychologia, vol. 40, no. 8, pp. 1313-1323, 2002.

[60] C. M. Walker, A. Sunderland, J. Sharma, and M. F. Walker, "The impact of cognitive impairment on upper body dressing difficulties after stroke: A video analysis of patterns of recovery," Journal of Neurology, Neurosurgery and Psychiatry, vol. 75, no. 1, pp. 43-48, 2004.

[61] S. Zinn, H. B. Bosworth, H. M. Hoenig, and H. S. Swartzwelder, "Executive function deficits in acute stroke," Archives of Physical Medicine and Rehabilitation, vol. 88, no. 2, pp. 173-180, 2007.

[62] Y. Stern, "Cognitive reserve and Alzheimer disease," Alzheimer Disease and Associated Disorders, vol. 20, no. 2, pp. 112-117, 2006.

[63] M. A. Ikram, H. A. Vrooman, M. W. Vernooij et al., "Brain tissue volumes in the general elderly population: the Rotterdam Scan Study," Neurobiology of Aging, vol. 29, no. 6, pp. 882-890, 2008.

[64] S. Das, N. Paul, A. Hazra et al., "Cognitive dysfunction in stroke survivors: a community-based prospective study from Kolkata, India," Journal of Stroke and Cerebrovascular Diseases, vol. 22, no. 8, pp. 1233-1242, 2013.

[65] C. Jacova, L. A. Pearce, R. Costello et al., "Cognitive impairment in lacunar strokes: the SPS3 trial," Annals of Neurology, vol. 72, no. 3, pp. 351-362, 2012.

[66] F. C. Goldstein, A. I. Levey, and N. K. Steenland, "High blood pressure and cognitive decline in mild cognitive impairment," Journal of the American Geriatrics Society, vol. 61, no. 1, pp. 6773, 2013.

[67] J. Birns and L. Kalra, "Blood pressure and vascular cognitive impairment: the debate continues," Journal of Human Hypertension, vol. 20, no. 1, pp. 1-3, 2006.

[68] M. Zhang, M. Chen, Q. Wang et al., "Relationship between cerebral microbleeds and cognitive function in lacunar infarct," Journal of International Medical Research, vol. 41, no. 2, pp. 347355, 2013.

[69] A. Tanaka, Y. Ueno, Y. Nakayama, K. Takano, and S. Takebayashi, "Small chronic hemorrhages and ischemic lesions in association with spontaneous intracerebral hematomas," Stroke, vol. 30, no. 8, pp. 1637-1642, 1999.

[70] R. P. Hart, J. B. Wade, and M. F. Martelli, "Cognitive impairment in patients with chronic pain: the significance of stress," Current Pain and Headache Reports, vol. 7, no. 2, pp. 116-126, 2003.

[71] A. Verdelho, S. Madureira, C. Moleiro et al., "Depressive symptoms predict cognitive decline and dementia in older people independently of cerebral white matter changes: the LADIS study," Journal of Neurology, Neurosurgery and Psychiatry, vol. 84, no. 11, pp. 1250-1254, 2013.

[72] O. Potvin, H. Forget, S. Grenier, M. Préville, and C. Hudon, "Anxiety, depression, and 1-year incident cognitive impairment in community-dwelling older adults," Journal of the American Geriatrics Society, vol. 59, no. 8, pp. 1421-1428, 2011.

[73] M. Martelli, N. Zasler, A. Mancini, and P. MacMillan, "Psychological assessment and applications in impairment and disability evaluations," in Guide to Functional Capacity Evaluation with
Impairment Rating Applications, R. V. May and M. F. Martelli, Eds., vol. 3, pp. 1-84, 1999.

[74] T. Pohjasvaara, M. Leskelä, R. Vataja et al., "Post-stroke depression, executive dysfunction and functional outcome," European Journal of Neurology, vol. 9, no. 3, pp. 269-275, 2002.

[75] F. Landi, G. Onder, M. Cesari et al., "Functional decline in frail community-dwelling stroke patients," European Journal of Neurology, vol. 13, no. 1, pp. 17-23, 2006.

[76] M. D. Patel, C. Coshall, A. G. Rudd, and C. D. A. Wolfe, "Cognitive impairment after stroke: clinical determinants and its associations with long-term stroke outcomes," Journal of the American Geriatrics Society, vol. 50, no. 4, pp. 700-706, 2002.

[77] M. F. M. Zulkifly, S. E. Ghazali, N. C. Din, and P. Subramaniam, "The influence of demographic, clinical, psychological and functional determinants on post-stroke cognitive impairment at day care stroke center, Malaysia," Malaysian Journal of Medical Sciences, vol. 23, no. 2, pp. 53-64, 2016. 


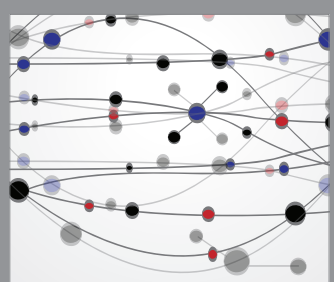

The Scientific World Journal
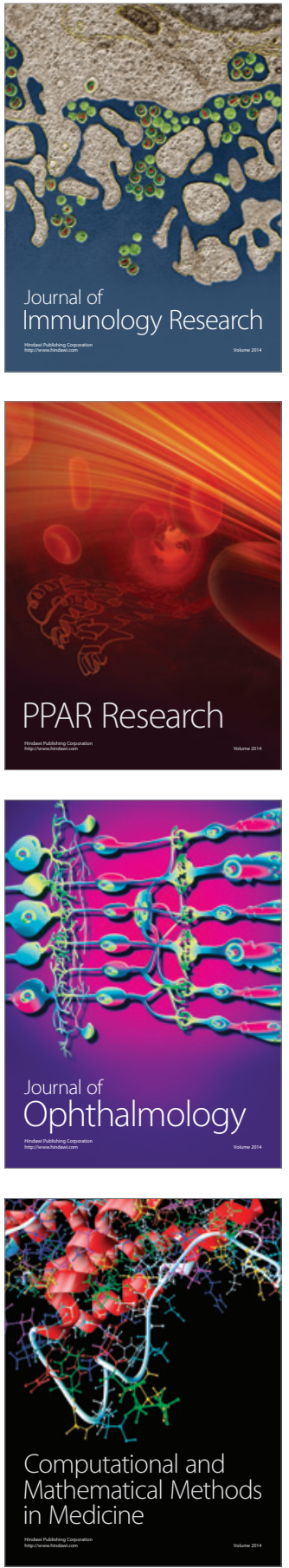

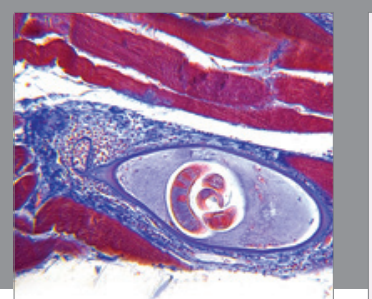

Gastroenterology Research and Practice

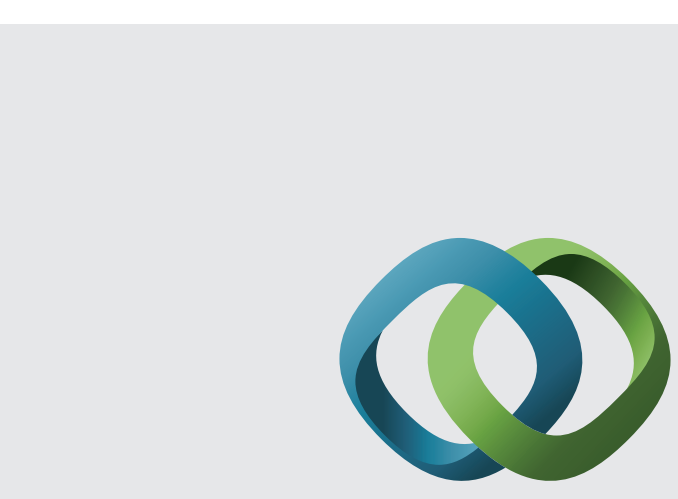

\section{Hindawi}

Submit your manuscripts at

http://www.hindawi.com
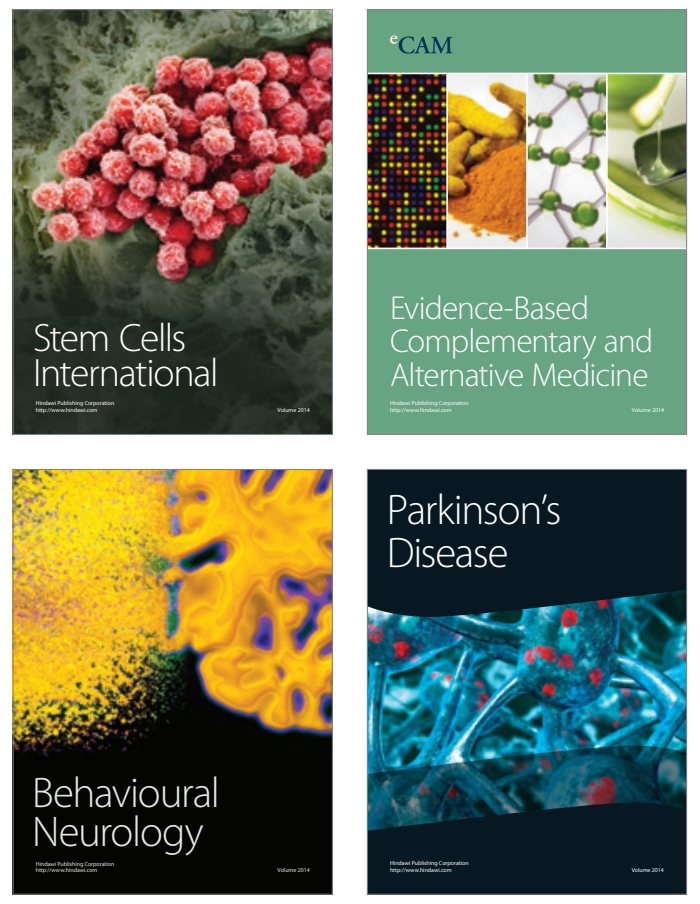
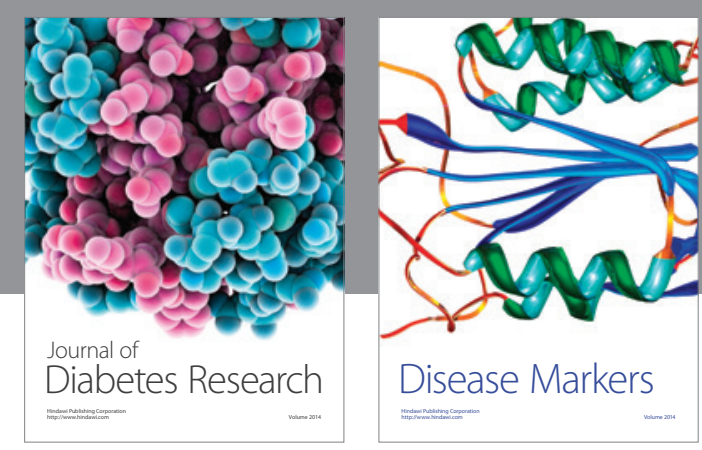

Disease Markers
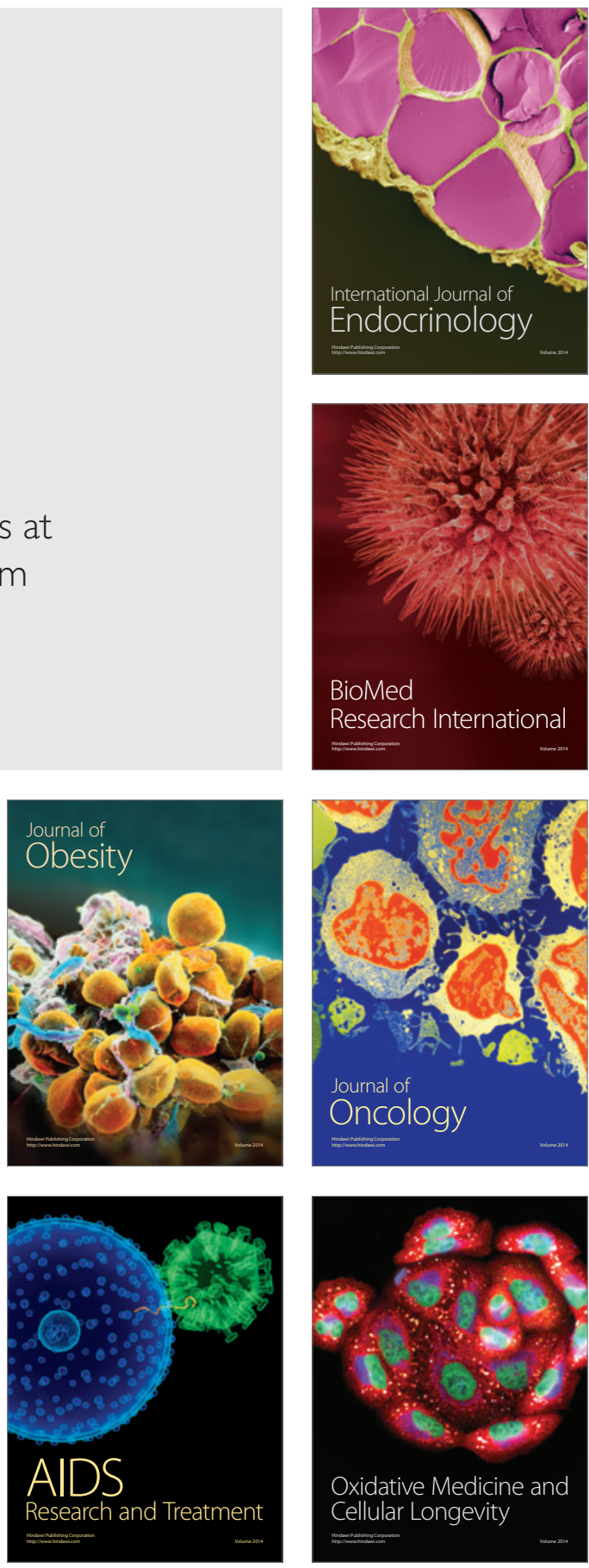